\title{
Dynamic plantwide modeling, uncertainty and sensitivity analysis of a pharmaceutical upstream synthesis: Ibuprofen case study
}

\author{
Montes, Frederico C. C.; Gernaey, Krist ; Sin, Gürkan
}

Published in:

Industrial and Engineering Chemistry Research

Link to article, DOI:

10.1021/acs.iecr.8b00465

Publication date:

2018

Document Version

Peer reviewed version

Link back to DTU Orbit

Citation (APA):

Montes, F. C. C., Gernaey, K., \& Sin, G. (2018). Dynamic plantwide modeling, uncertainty and sensitivity analysis of a pharmaceutical upstream synthesis: Ibuprofen case study. Industrial and Engineering Chemistry Research, 57(30), 10026-10037. https://doi.org/10.1021/acs.iecr.8b00465

\section{General rights}

Copyright and moral rights for the publications made accessible in the public portal are retained by the authors and/or other copyright owners and it is a condition of accessing publications that users recognise and abide by the legal requirements associated with these rights.

- Users may download and print one copy of any publication from the public portal for the purpose of private study or research.

- You may not further distribute the material or use it for any profit-making activity or commercial gain

- You may freely distribute the URL identifying the publication in the public portal 


\title{
Process Systems Engineering
}

\section{Dynamic plantwide modeling, uncertainty and sensitivity analysis of a pharmaceutical upstream synthesis: Ibuprofen case study}

\author{
Frederico Montes, Krist V. Gernaey, and Gurkan Sin
}

Ind. Eng. Chem. Res., Just Accepted Manuscript • DOI: 10.1021/acs.iecr.8b00465 • Publication Date (Web): 17 May 2018

Downloaded from http://pubs.acs.org on May 25, 2018

\section{Just Accepted}

"Just Accepted" manuscripts have been peer-reviewed and accepted for publication. They are posted online prior to technical editing, formatting for publication and author proofing. The American Chemical Society provides "Just Accepted" as a service to the research community to expedite the dissemination of scientific material as soon as possible after acceptance. "Just Accepted" manuscripts appear in full in PDF format accompanied by an HTML abstract. "Just Accepted" manuscripts have been fully peer reviewed, but should not be considered the official version of record. They are citable by the Digital Object Identifier (DOI®). "Just Accepted" is an optional service offered to authors. Therefore, the "Just Accepted" Web site may not include all articles that will be published in the journal. After a manuscript is technically edited and formatted, it will be removed from the "Just Accepted" Web site and published as an ASAP article. Note that technical editing may introduce minor changes to the manuscript text and/or graphics which could affect content, and all legal disclaimers and ethical guidelines that apply to the journal pertain. ACS cannot be held responsible for errors or consequences arising from the use of information contained in these "Just Accepted" manuscripts. 


\title{
Dynamic plantwide modeling, uncertainty and
} sensitivity analysis of a pharmaceutical upstream synthesis: Ibuprofen case study

\author{
Frederico C. C. Montes, Krist Gernaey, Gürkan Sin* \\ PROSYS, Department of Chemical and Biochemical Engineering, Technical University of \\ Denmark, Søltofts Plads, Building 229, 2800 Kgs. Lyngby, Denmark \\ * Corresponding author: gsi@,kt.dtu.dk
}

KEYWORDS: Process modelling, Ibuprofen synthesis, uncertainty and sensitivity analysis

\begin{abstract}
A dynamic plantwide model was developed for the synthesis of the Active Pharmaceutical Ingredient (API) ibuprofen, following the Hoescht synthesis process. The kinetic parameters, reagents, products and by-products of the different reactions were adapted from literature, and the different process operations integrated until the end process, crystallization and isolation of the ibuprofen crystals. The dynamic model simulations were validated against available measurements from literature and then used as enabling tool to analyze the robustness of design space. To this end, sensitivity of the design space towards input disturbances and process
\end{abstract}


uncertainties (from physical and model parameters) is studied using Monte Carlo simulations. The results quantify the uncertainty of the quality of product attributes, with particular focus on crystal size distribution and ibuprofen crystalized. The ranking of the most influential parameters on the chosen quality attributes is presented, with crystal growth and water concentration being the most influential ones. The total amount of saturated solvent, which propagates from upstream processes, has been shown to highly influence the total mass of crystal produced, and the underspecified API as well. This dynamic plantwide modeling coupled with Monte Carlo simulations is valuable to improve design and optimization of pharmaceutical processes at early stages, especially to bottleneck the design space against a range of uncertainties and disturbances. 


\section{Introduction}

The pharmaceutical industry is continuously under pressure. While the competition with generic drug manufacturers is as fierce as ever, regulations are increasingly strict, and growing awareness that drug manufacturing can have a significant environmental impact leads to growing demand from the consumers to environmentally friendly pharmaceutical production, or greener products ${ }^{[1,2]}$. In addition, development costs for new drugs are somewhere between a couple of hundreds of thousands of dollars to a few billions ${ }^{[3,4]}$, and this cost value has been shown to steadily annual increase $^{[5]}$. Facing these conditions, and with a fixed patent lifetime, modern pharmaceutical industry needs to make an effort in evolving towards more efficient and innovative production processes.

This has been particularly stressed by the U.S. Food and Drug Administration (FDA), with the introduction of Process Analytical Tools (PAT) concept ${ }^{[6]}$. Within the referred document, the FDA encouraged the usage of different tools for process understanding, design space exploration, and online monitoring and control. Special emphasis was given to the use of multivariate tools for design, data acquisition and data analysis. As defended by other studies ${ }^{[2]}$, Process Systems Engineering (PSE) methods are critical for these achievements, and these methods have been broadly used by other industries (petrochemical and polymer industry for example) ${ }^{[7]}$, as well as increasingly applied by bio-based industries ${ }^{[8]}$. Whether the objective is to evaluate and improve an existing API synthesis process, or study and analyze different operability spaces and/or different flowsheet hypothesis, many PSE methods have been studied and improved over time ${ }^{[2]}$. For instance, different authors developed tools for the generation and evaluation of different pharmaceutical process designs with ecological considerations ${ }^{[9-11]}$. By integrating these with component's physical properties databases, pollution prevention is focused from the early stages of 
process development and intensification, instead of focusing in waste treatment solutions at the end of the process. Furthermore, through the application of mechanistic models it has been shown that continuous pharmaceutical manufacturing can achieve low environmental footprints ${ }^{[12]}$ and, as demonstrated, the continuous manufacturing of an API can achieve lower total costs, when compared to batch production of the same components ${ }^{[13,14]}$. Addressing uncertainties in the process, either by a mechanistic model description or experimental approaches ${ }^{[15,16]}$, enables probabilistic risk analysis. This allows a process engineer to understand the chances of not achieving the critical quality attributes (CQA) of a certain process, i.e., the scenario of failing to meet target design specs and/or the scenario of being below the target process economic metrics (e.g. certain net present value, as demonstrated elsewhere ${ }^{[17]}$ ). The study of the possible scenarios helps in long term operation planning and to make solid and decisive decisions when the whole process/market is uncertain. Different studies and efforts have focused on methodologies ${ }^{[18]}$ and tools to help and support such cases, while addressing economic and ecological objectives ${ }^{[11,19]}$, and including future possible changes in the regulatory policies for waste management and treatment ${ }^{[19]}$. Further including sensitivity analysis, either local or global ${ }^{[15,20]}$, is definitely essential for experimental design and focus of collecting critical missing or misleading data. In addition, the identification of most the influential parameters of a system is beneficial for the implementation of new and more advanced control strategies. These strategies gained a new focus in the past years in the pharmaceutical industry, with downstream hybrid MPC-PID control implementations to reduce the final product off-spec due to upstream disturbances/uncertainty ${ }^{[21]}$, model predictive control of complex batch processes (such as crystallization) ${ }^{[22-24]}$, amongst others.

One API in particular has had quite the attention from academia in the past years: Ibuprofen (2(4-isobutylphenyl) propionic acid). This commonly known analgesic was considered an essential drug by the World Health Organization in 2009, even thought it was first synthetized by Boots in 
the 1960s. The Boots synthesis method was overcome by the Hoechst process, which reduced the number of synthesis steps from six to three. Although nowadays there are more efficient synthesis routes $^{[25]}$ and efforts are being made to show that moving from batch production to continuous is viable ${ }^{[14,26]}$, much of the on-going industry still relies on the Hoechst batch process. However, and to our best knowledge, there has been no focus in studying the design-space of the upstream synthesis, even though that data and reaction kinetic studies have been published in some of the synthesis steps, taking into consideration the choice of catalysts, process pressure and temperature [27-29].

In this work, the objective is to develop a dynamic plantwide model for simulation, uncertainty and sensitivity analysis of the upstream process of ibuprofen through the Hoechst synthesis path. The dynamic flowsheet model is based on the available data regarding the process, and the operability space of each individual model is the same as the published one. Several assumptions were made, regarding some intermediate properties and purification steps. The design space is explored through a sensitivity analysis and error propagation performed on parameters such as each model temperature, pressure and $\mathrm{pH}$. After exploring the impact they have further downstream, a detailed population balance cooling crystallization model is included. A global sensitivity analysis and Monte Carlo based nonlinear error propagation of upstream process parameters, models parameters and physical parameters is then applied, and deviations in the crystal size distribution and crystal produced are ranked accordingly to the influence from the input analysis. The outcome opens the to further plantwide control implementation, and directs towards experimental design.

This paper is organized as follows: first the case study and methodology is presented in the material and methods section. The methodology consists of : (i) model collection and analysis, (ii) flowsheet assembly and dynamic simulation, and (iii) Uncertainty and sensitivity analysis. In the results and discussion section, first model validation results for selected unit operations are given 
followed by model integration and dynamic simulation results for cyclic steady state. These are followed by local sensitivity analysis in the upstream synthesis of the process parameters to study influence of process parameters. Finally global uncertainty and sensitivity analysis results obtained from crystallization process are presented to assess the impact of error propagation on the critical attributes of product. The paper ends with concluding remarks and perspectives.

\section{Material and methods}

The ibuprofen synthesis case study is based on the Hoescht path ${ }^{[30]}$, which consists of three main reaction steps: Friedels craft acetylation of isobutylbenzene into 4-isobutyl acetophenone (IBAP), hydrogenation of IBAP into 1-(4-isobutylphenyl)ethanol (IBPE) in the presence in the presence of a catalyst, and carbonylation of the produced IBPE into ibuprofen and by-products in the presence of a catalyst. In this work, as no data has been found for the first reaction, IBPA is assumed to be the initial reagent in this chain. The referred process steps are discontinuous, and were assumed occur in isothermal conditions and in perfectly mixed batch vessels.

\subsection{Model collection and analysis}

The reaction kinetics, solvents and by-products are adapted from elsewhere ${ }^{[28]}$ for the hydrogenation synthesis:

$$
\begin{aligned}
& \frac{d C_{I B A P}}{d t}=-r_{h, 1}==\frac{C_{c a t} a k_{h, 1} C_{I B A P} P_{H 2}}{\left(1+K_{I B A P} C_{I B A P}+\sqrt{K_{h} P_{H 2}}+K_{H 2 O} C_{H 2 O}\right)^{2}} \\
& \frac{d C_{I B P E}}{d t}=r_{h, 1}-r_{h, 2}==r_{h, 1}-\frac{C_{c a t} a k_{h, 2} C_{I B P E} P_{H 2}}{\left(1+K_{I B A P} C_{I B A P}+\sqrt{K_{h} P_{H 2}}+K_{H 2 O} C_{H 2 O}\right)^{2}} \\
& \frac{d C_{\text {olig }}}{d t}=r_{h, 3}==\frac{C_{c a t} a k_{h, 3} C_{I B P A}^{2}}{\left(1+K_{I B A P} C_{I B A P}+\sqrt{K_{h} P_{H 2}}+K_{H 2 O} C_{H 2 O}\right)^{2}} \\
& \frac{d C_{I B E B}}{d t}=r_{h, 2} \\
& a=e^{\left(-\alpha \frac{C_{\text {olig }}}{C_{c a t}}\right)}
\end{aligned}
$$


where $C_{I B E B}$ represents the molar concentration of the by-product 4-isobutylethylbenzene (IBEB) and $C_{H 20}$ represents the molar concentration of water. Water formation is proportional to the second reaction (eq.4). Rate and equilibrium constants are used after the same work:

$k_{h, i(T)}=k_{h, i\left(T_{0}\right)} e^{\left(-\frac{E a_{h y d, i}}{R}\left(\frac{1}{T}-\frac{1}{T_{0}}\right)\right)}$

The kinetics, solvents and by-products for the carbonylation step are also adapted from literature $^{[29]:}$

$\frac{d C_{I B P E}}{d t}=-r_{c, 1}=-k_{c, 1} C_{I B P E} C_{H+}$

$\frac{d C_{I B S}}{d t}=r_{C, 1}-r_{C, 2}+r_{\mathrm{c},-2}=r_{\mathrm{c}, 1}-k_{\mathrm{c}, 2} C_{I B S} C_{H+} C_{C l-}+k_{\mathrm{c},-2} C_{I B P C l}$

$\frac{d C_{I B P C l}}{d t}=r_{\mathrm{c}, 2}-r_{\mathrm{c},-2}+r_{\mathrm{c}, 3}=r_{\mathrm{c}, 2}-r_{\mathrm{c},-2}-\frac{k_{\mathrm{c}, 3} C_{I B P C l} \mathrm{C}_{c, H 2 O} \mathrm{C}_{C O} C_{c, c a t}{ }^{0.43}}{1+K_{C} C_{I B P C l}}$

$\frac{d C_{\text {prod }}}{d t}=r_{c, 3}$

where $C_{I B S}, C_{I B P C l}, C_{\text {prod }}$ and $C_{I B P E}$ represent respectively the molar concentration of 4 -isobutylstyrene (IBS), the molar concentration of 1-(4-iso-butylphenyl)ethyl chlorine (IBPCl), the molar concentration of the end-products IBU and 3-(4-iso-butylphenyl)propionic acid (3-IPPA), and the molar concentration of IBPE. The carbonylation kinetic parameters are calculated by equation 11, and the concentration of carbon monoxide dissolved $\left(C_{C O}\right)$ is calculated through equation 12:

$k_{c, i}=k_{0, i} e^{\frac{-E a_{c, i}}{R * T}}$

$C_{C o}=P_{c o} H_{e}$

where Pco is the partial pressure of $\mathrm{CO}$, and $H_{e}$ is the Henry's constant of $\mathrm{CO}$ in a mixture of IBPE and the reaction's solvent, methyl-ethyl-ketone (MEK). The dependence of $H_{e}$ with the 
weight ratio of IBPE in MEK and the mixture temperature has been reported ${ }^{[28]}$. A linear model was used to generate the $H_{e}$ values depending on the IBPE mass ration and the process temperature, and can be consulted in Supporting Information (section A).

The model used for the ibuprofen cooling crystallization is a population balance model based on the method of classes, adapted from elsewhere ${ }^{[31]}$. For a two dimension crystallization problem, the number of elements ( $N$ crystals) in in the $i^{\text {th }}$ class of the first characteristic length and in the $j^{\text {th }}$ class of the second characteristic length is described as:

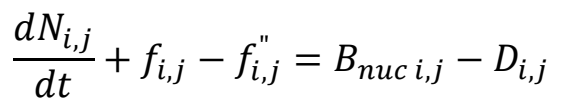

where $f$ and $f$ " represent the inflow from and outflow to adjacent classes, $B_{\text {nuc }}$ is the term of birth (newly formed crystals due to nucleation) and $D$ commonly stands for term death of crystals (phenomena in the crystallization that include breakage of the crystals). The inflows and outflows of the adjacent classes are illustrated by the equation (14).

$f_{i, j}=\frac{G_{x, i-1} N_{i-1, j}}{2 * C_{x(i-1)}}+\frac{G_{y, j-1} N_{i, j-1}}{2 * C_{y(j-1)}}$

where $C_{x}$ and $C_{y}$ represent the discretization space, or the classes. The growth kinetics of ibuprofen in an aqueous-ethanol solution are adapted from elsewhere ${ }^{[32-34]}$. Equations 15 to 19 represent, respectively, the growth value of crystals, the super-saturation curve, the saturation profile, the temperature inside the crystallizer and the temperature of the cooling serpentine:

$G_{i}=k_{i} S_{\text {sat }}{ }^{n}$

$S_{\text {sat }}=\max \left(0, \frac{C_{i b u}-C_{\text {sat }}}{C_{\text {sat }}}\right)$

$C_{\text {sat }}=0.495+0.001026 T_{\text {cryst }}^{2}$

$\frac{d T_{\text {cryst }}}{d t}=\frac{-\Delta H_{c} \rho_{c} V_{\text {form }, \text { cryst }}-U A\left(T_{\text {cryst }}-T_{w}\right)}{\rho_{\text {sol }} V c_{p, \text { sol }}}$ 
$\frac{d T_{w}}{d t}=\frac{F_{w, \text { in }} \rho_{\text {water }} c_{p, \text { water }}\left(T_{w, \text { in }}-T_{w}\right)+U A\left(T_{\text {cryst }}-T_{w}\right)}{\rho_{\text {water }} V_{\text {water }} c_{p, \text { water }}}$

Where $\Delta H_{c}$ is the enthalpy of crystallization, $\rho_{c}$ the crystal density, $V_{\text {form,cryst }}$ is the volume of newly formed crystal, $\rho_{\text {sol }}$ is the density of the solution, $c_{p, s o l}$ is the specific heat capacity of the solution, $V$ is the solution volume, $F_{w, i n}$ is the inflow of cooling water in the cooling serpentine, $\rho_{\text {water }}$ is the density of water, $c_{p, \text { water }}$ is the specific heat capacity of water, $T_{w, i n}$ is the inlet temperature of the cooling water, $U$ is the Heat transfer coefficient of the crystallizer wall, and $\mathrm{A}$ is the surface area of the cooling serpentine. Values were taken from literature ${ }^{[23,35]}$. The temperature within the crystallizer, $T_{\text {cryst }}$, depends on the cooling profile imposed to the cooling serpentine, $T_{w}$, by changing the temperature of the water inflow. The water inflow temperature was assumed to be composed of two profiles, described by equation (20):

$$
\left\{\begin{array}{cl}
T_{\text {max }}-\frac{t\left(T_{\text {max }}-T_{\text {lin }}\right)}{t_{\text {linear }}}, & t<t_{\text {linear }} \\
T_{\text {lin }}-\left(T_{\text {lin- }} T_{\min }\right)\left(\frac{t-t_{\text {linear }}}{t_{\text {max }}-t_{\text {linear }}}\right)^{1.5}, & t>t_{\text {linear }}
\end{array}\right.
$$

where $T_{\max }$ is the initial temperature of the crystallization, $T_{\text {lin }}$ is the end temperature of the linear decrease, $t_{\text {linear }}$ is the time of linear decrease, $T_{\min }$ is the end temperature of the crystallization, and $t_{\max }$ is the total duration of the crystallization. The remaining assumptions done for the crystallization can are present in the Supporting Information (section B).

Both reaction models (carbonylation and hydrogenation) where compared against the published data from the same authors ${ }^{[28,29]}$. This was done by fitting the parameters to the data reported minimizing the sum of the squared errors, and verifying if the estimated value would coincide within the $95 \%$ confidence interval reported. If the estimated parameters fit within the reported intervals, no further work is performed on the model, and the reported parameters are used for the 
upcoming tasks. However, if this is not the case, a parameter estimation, identifiability analysis and uncertainty analysis are performed.

- For the parameter estimation, the methodology follows the workflow of Sin et al. ${ }^{[36]}$, using the maximum likelihood estimation method from Seber \& Wild ${ }^{[37]}$ where the covariance matrix $\operatorname{COV}(\theta)$, can be estimated by eq. 20 :

$$
\begin{aligned}
& \operatorname{COV}(\theta)=\frac{J(\theta)}{N-p}\left(\left(\frac{d y}{d \theta}\right)^{T} Q_{m}^{-1}\left(\frac{d y}{d \theta}\right)\right) \\
& J(\theta)=\arg \min \sum_{i}\left(y_{i}^{e x p}-y_{i}^{\text {pred }}\right)^{2}
\end{aligned}
$$

Where $J(\theta)$ is the minimum sum of squared errors obtained from the least-squares parameter estimation (eq. 21), $\frac{d y}{d \theta}$ is the sensitivity matrix of the model variables (y) to the parameters $(\theta), \mathrm{Q}_{\mathrm{m}}$ is the covariance matrix of measurement errors, and $N$ and $p$ are the total number of measurements and the total number of estimated parameters, respectively. The correlation between two parameters is then calculated as it follows:

$$
\operatorname{CoR}\left(\theta_{z}, \theta_{w}\right)=\frac{\operatorname{Cov}\left(\theta_{z}, \theta_{w}\right)}{\sqrt{\sigma_{\theta_{z}}^{2} \sigma_{\theta_{w}}^{2}}}
$$

- For identifiability analysis, the two step procedure of Brun and co-workers ${ }^{[38]}$ as described in Sin. et al. ${ }^{[36]}$ was used. The identifiability method ranks the parameter significance, $\delta^{\text {msqr }}$, and a collinearity analysis, $Y_{k}$, are obtained though eqs. 23-26:

$$
\begin{aligned}
& \delta^{m s q r}=\sqrt{\frac{1}{N} \sum_{i}^{N} s r_{i}} \\
& Y_{k}=\frac{1}{\sqrt{\min \left(\lambda_{K}\right)}}
\end{aligned}
$$


$\lambda_{K}=\operatorname{eigen}\left(\operatorname{snorm}_{K}^{T} \operatorname{snorm}_{K}\right)$

snorm $=\frac{s r}{\|s r\|}$

where $s r$ is the vector of non-dimensional sensitivity values, $K$ a parameter subset, $\lambda_{K}$ is the eigen values of the normalized sensitivity matrix for parameter subset $K$, and snorm is the normalized non-dimensional sensitivity function using Euclidian norm. The collinearity index is used to find an appropriate subset of parameters with a value between 10 and 15, as largely used in literature ${ }^{[38,36]}$

The uncertainty analysis performed is based on the well-defined Bootstrap method ${ }^{[39]}$, and the methodology can be found in the work of Frutiger et al. ${ }^{[40]}$.

\subsection{Flowsheet assembly and dynamic simulation}

In order to simulate the dynamic behavior of the ibuprofen synthesis, the previous described models are integrated. To connect the discontinuous (batch type) unit processes with the continuously operated operations, several simplified models were included, such as buffer tanks (before and after each major process step), a simplified decanter for the reaction steps (based on available information ${ }^{[30]}$ ), and assumed distillation fractions based on predicted boiling points of the involved components (the boiling points were obtained using Group-Contribution based property estimation method $\left.{ }^{[41]}\right)$. The predicted values are presented in Supporting Information (section C, table S.2).

The holdup within the buffer tanks is described by equation (27). The valve values change between 0 and 1 , depending on the operating phase of the previous or following unit operation. For instance, the outlet valve of the buffer tank preceding the hydrogenation step follows equation (28): 
$\frac{d V_{i}}{d t}=$ valve $_{i n, i} F_{\text {in, },}-$ valve $_{\text {out }, i} F_{\text {out }, i}$

valve $_{\text {out } \text {,pre-cryst }}\left\{\begin{array}{c}1, \quad t_{\text {operation }}<t_{\text {hydrogenaion reaction }} \\ 0, \text { otherwise }\end{array}\right.$.

$t_{\text {operation }}$ is a cyclic value between 0 and the sum of the emptying time, filling time and reaction time of the respective operation. The value resets to 0 when the max value is achieved. The integration was performed in a Matlab/Simulink interface (The MathWorks ${ }^{\circledR}$, Natick, MA), divided in three steps:

- The first step is resumed into the user input: this is performed in Matlab, where the initial conditions, batch time, emptying and filling time, and process parameters are chosen. Process understanding is the most important key in this part, as a huge number of possibilities exist for process input. However, the user needs to pay close attention to the feasibility of those choices. One common example, is the constant increase in the tanks holdup content, or running dry;

- The second step is performed within Simulink, where the simulation occurs until the filling of the crystallizer. This is due to the fact that for the simulation of the initial models a fixed step solver was found to give a faster result (ode4 Runge-Kutta). The results are saved and sent to the third step;

- The third step is simulation of the ibuprofen crystallization. Extra inputs from the user are requested, such as the initial seed mass and size characteristics, and the cooling profile. Ode $15 \mathrm{~s}$ is the solver used for this population balance model.

The dynamic flowsheet model implemented in Matlab Simulink is displayed in figure 1.

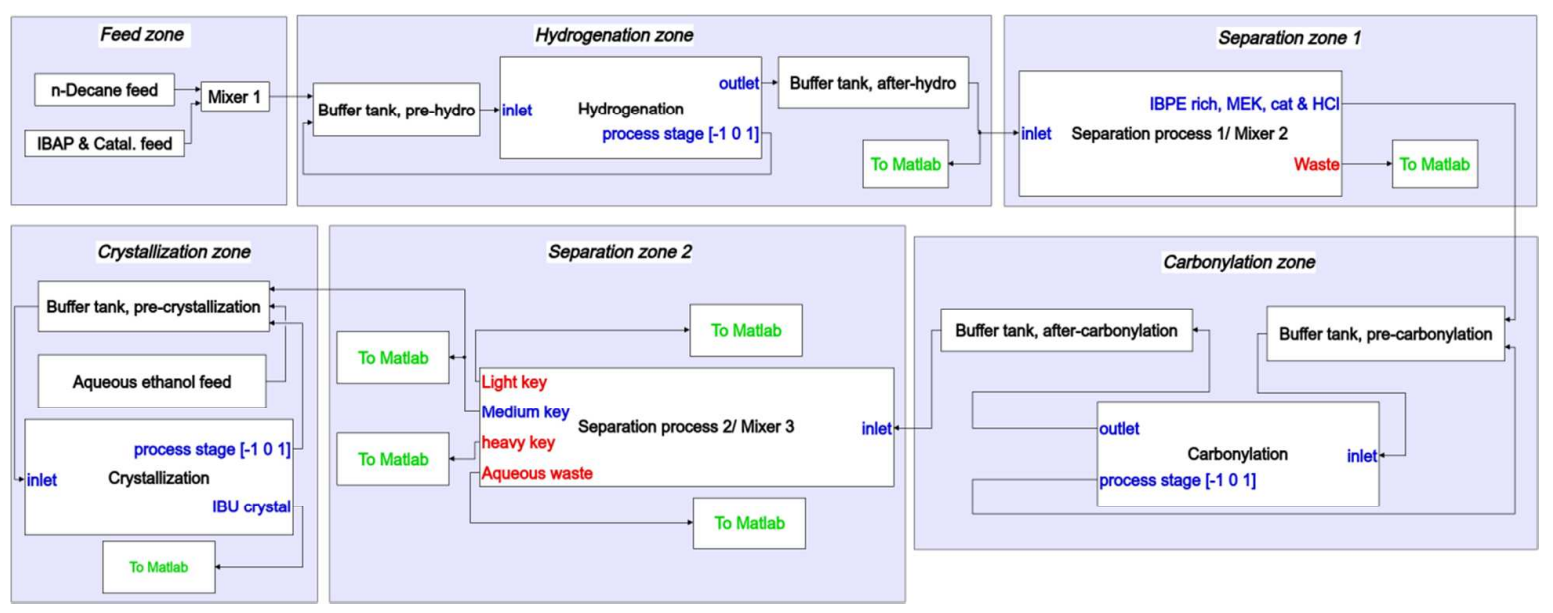


Figure 1 - Dynamic flowsheet model implementation in Matlab Simulink

\subsection{Uncertainty and sensitivity analysis}

Cyclic-steady state is reached globally, with each dynamic state being periodic. Hence, the design space can be studied with disturbances to the system, and the weight of those disturbances analysed. Therefore, local sensitivity analysis was performed by changing key parameters, one factor at a time, in accordance to other authors ${ }^{[15]}$. These are available in Supporting Information (table S.3, section D), together with the deviation from the initial value. The propagation of the deviation is then carried out to the final crystallization model. Here, uncertainty analysis and global sensitivity analysis were performed. For uncertainty analysis, we used the engineering standard technique of Monte Carlo method ${ }^{15]}$, consisting of three steps: (1) specifying input uncertainty, (2) sampling input uncertainty, and (3) propagating the sample input uncertainty through the model, obtaining different output vector.

\footnotetext{
(1) The input uncertainty was defined as a uniform distribution centralized in the parameter mean, $\theta_{\text {mean }}$, with maximum and minimum values being calculated as follows:

$\theta_{\min }=(1-\%$ Variation $) * \theta_{\text {mean }}$

$\theta_{\text {max }}=(1+\%$ Variation $) * \theta_{\text {mean }}$

(2) The probabilistic sampling of the input, Latin Hypercube Sampling was used ${ }^{[42]}$, with 300 samples from the input space defined in (1). No correlation was assumed for the samples, as no previous information was available. 
(3) The propagation of the sampling space through the dynamic model was done over the 5 hours crystallization, with data collected every minute (300 points) for the CSD. The uncertainty of the model outputs for the final simulation time is represented with mean and $95 \%$ confidence interval.

For global sensitivity analysis we used linear regression of Monte Carlo outputs, so called Stadardised Regression Coefficients (SCR). The scalar model output matrix can be denoted as sy and has the dimension of $K^{*} N$, where $K$ is the number of output variables and $N$ the number of samples. The regression model is then fitted to the scalar output of the Monte Carlo simulations relating model output, $s y$ to the model inputs considered in the uncertainty analysis, $\theta_{i j}$ as shown in equation (31):

$s y_{i k}=b_{0 k}+\sum_{j=1}^{M} b_{j k} \theta_{i j}+\varepsilon_{i k}$

Where $s y_{i k}$ is the scalar value for the $k^{t h}$ output, $b_{j k}$ is the coefficient of the $j^{\text {th }}$ input parameter, $\theta_{j}$, for the $k^{\text {th }}$ output, $\theta_{i j}$ is the value of the $j^{\text {th }}$ parameter and $\varepsilon_{i k}$ is the error of the regression model. Equation (30) is then written in dimensionless form by scalling the outputs and the parameters using their corresponding mean and standard deviations ${ }^{[43]}$ as expressed in equation (32):

$\frac{s y_{i k}-\mu_{s y_{k}}}{\sigma_{s y_{k}}}=\sum_{j=1}^{M} \beta_{j k} \frac{\theta_{i j}-\mu_{\theta_{j}}}{\sigma_{\theta_{j}}}+\varepsilon_{i k}$

The standardized regression coefficients, $\beta_{j k}$, can range from [-1 1]. A negative sign represents a negative effect in the output, and vice-versa, and its magnitude defines the influence of the input on the model output. 


\section{Results and discussion}

Model Validation. The data fitting of the hydrogenation parameters agreed with the results presented by Thakar et al. ${ }^{[28]}$, and can be observed in figure 2 . The estimated parameters were obtained through a minimization of the squared errors between the reported data and model predictions. As the new estimations are within the reported confidence interval, no further work is performed. The results can be observed in table 1 .

Table 1 - Comparison between reported and calculated kinetic parameters for hydrogenation

\begin{tabular}{|c|c|c|c|c|c|}
\hline \multicolumn{2}{|c|}{ Parameter } & \multicolumn{3}{|c|}{ Reported values } & \multirow[t]{3}{*}{ Estimated values } \\
\hline & & & \multicolumn{2}{|c|}{$\begin{array}{l}\text { Values at } 95 \% \\
\text { confidence interval }\end{array}$} & \\
\hline & & & $-95 \%$ & $+95 \%$ & \\
\hline $\mathrm{k}_{\mathrm{h}, 1}$ & $\mathrm{~L} /\left(\mathrm{g}_{\text {cat }}\right.$ atm s $)$ & 1.14 & 0.89 & 1.39 & 1.22 \\
\hline $\mathrm{k}_{\mathrm{h}, 2}$ & $\mathrm{~L} /\left(\mathrm{g}_{\text {cat }}\right.$ atm s $)$ & 0.095 & 0.075 & 0.115 & 0.088 \\
\hline $\mathrm{k}_{\mathrm{h}, 3}$ & $\mathrm{~L}^{2} /\left(\mathrm{g}_{\text {cat }}\right.$ atm $\left.\mathrm{s}\right)$ & 0.024 & 0.020 & 0.028 & 0.021 \\
\hline $\mathrm{K}_{\mathrm{IBAB}}$ & $\mathrm{L} / \mathrm{mol}$ & 76.4 & 52.7 & 100.1 & 69.5 \\
\hline $\mathrm{K}_{\mathrm{H} 2 \mathrm{O}}$ & $\mathrm{L} / \mathrm{mol}$ & 529 & 420 & 635 & 634 \\
\hline
\end{tabular}

However, there was a full disagreement with the reported values in literature ${ }^{[29]}$, for the carbonylation of IBPE. This mainly due to the lack of understanding of the initial concentration for 
the hydrochloric acid. Assuming a constant concentration of hydrochloric acid $(\mathrm{HCl})$ of $0.24 \mathrm{~mol} / \mathrm{L}$ and $2.67 \mathrm{~mol} / \mathrm{L}$ of water, a parameter re-estimation, identifiability analysis and uncertainty analysis was performed for the carbonylation process.

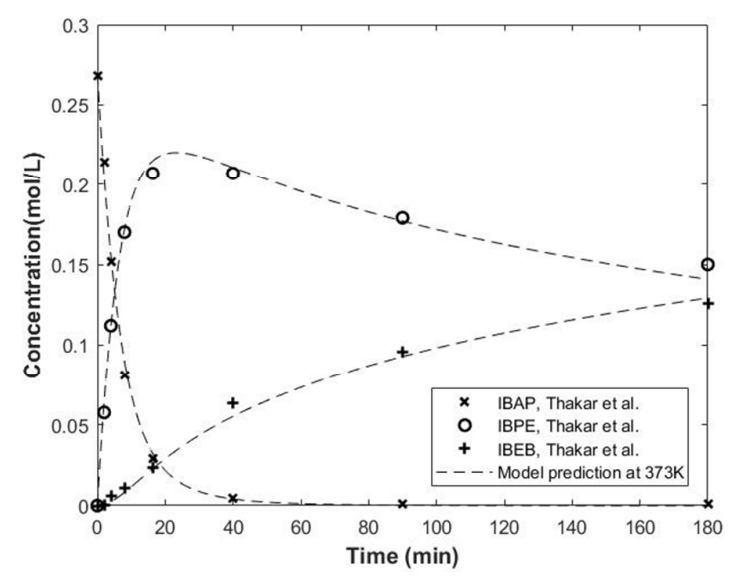

Figure 2 - Comparison between the reported experimental values at $373 \mathrm{~K}$

It can be observed in table 2 that the available data ${ }^{[29]}$ is not information-wise rich enough to obtain a unique set of kinetic parameters values that represent the system, using the model presented in equation (7-11). In fact, the correlation matrix in Supporting Information (table S.4 section E) shows that there is a huge correlation between the kinetic parameters and their respective activation energies.

Table 2 - Parameter fitting from the carbonylation literature

\begin{tabular}{|c|c|c|c|c|c|c|c|c|c|}
\hline & $E a_{c, 1}$ & $E a_{c, 2}$ & $E a_{c,-2}$ & $E a_{c, 3}$ & $k_{0,1}$ & $k_{0,2}$ & $k_{0,-2}$ & $k_{0,3}$ & $K_{c}$ \\
\hline Units & $\mathrm{kJ} / \mathrm{mol}$ & $\mathrm{kJ} / \mathrm{mol}$ & $\mathrm{kJ} / \mathrm{mol}$ & $\mathrm{kJ} / \mathrm{mol}$ & $\begin{array}{c}/(m o l . \\
s)\end{array}$ & $L^{2} /\left(\mathrm{mol}^{2} . s\right)$ & $L /($ mol.s $)$ & $\begin{array}{c}L^{2.43} /\left(\mathrm{mol}^{2} .\right. \\
\left.{ }^{43} . \mathrm{s}\right)\end{array}$ & $\mathrm{L} /(\mathrm{mol})$ \\
\hline $\begin{array}{l}\text { Estimated } \\
\text { value }\end{array}$ & 21.43 & 43.51 & 35.52 & 35.14 & 6.07 & $1.38 \mathrm{E}+04$ & $1.52 \mathrm{E}+02$ & $1.64 \mathrm{E}+05$ & 26.57 \\
\hline
\end{tabular}




\begin{tabular}{|l|c|c|c|c|c|c|c|c|c|}
\hline$\sigma$ & 2.50 & 21.77 & 66.42 & 4.82 & 4.71 & $9.34 \mathrm{E}+04$ & $3.13 \mathrm{E}+03$ & $2.48 \mathrm{E}+05$ & 14.26 \\
\hline$-95 \%$ & 16.46 & 0.24 & -96.50 & 25.56 & -3.29 & $-1.72 \mathrm{E}+05$ & $-6.07 \mathrm{E}+03$ & $-3.29 \mathrm{E}+05$ & -1.78 \\
\hline$+95 \%$ & 26.41 & 86.77 & 167.54 & 44.72 & 15.426 & $1.99 \mathrm{E}+05$ & $6.38 \mathrm{E}+03$ & $6.56 \mathrm{E}+05$ & 54.92 \\
\hline
\end{tabular}

Following the methods for identifiability analysis described prior to this section, the ranking of the parameter significance can be observed in figure 3 .

Apart from the IBPE plot (which is only influenced by eq. (7) activation energy and reaction rate), it can be observed that the activation energies have the most impact in the outcome of the system. This has been taken into consideration when choosing a suitable subset (out of 502 possible combinations) for a new parameter estimation. Regarding the collinearity analysis, subsets consisting of 5 parameters in which 4 are the activation energies, are within the gap used in literature $^{([38,36])}$
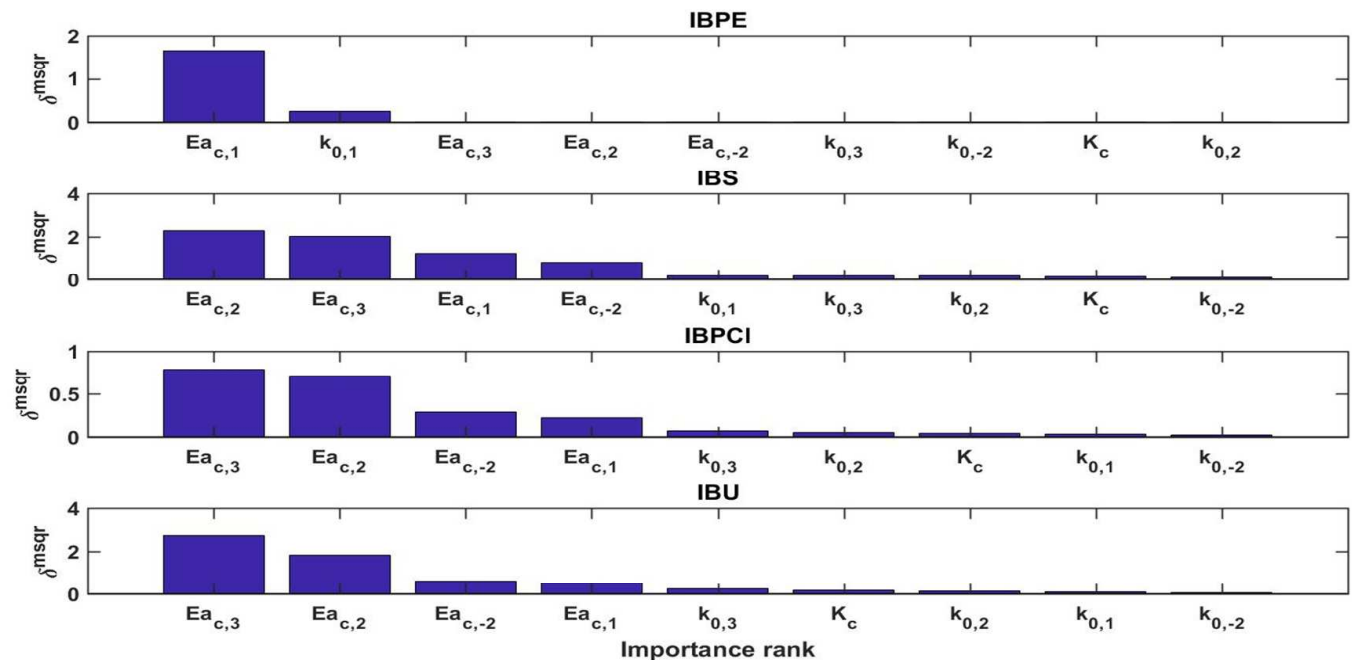

Figure 3 - Parameter significance ranking for the carbonylation model

However, the correlation and uncertainty obtained was still non-reasonable and very high. The subset consisting of the 4 activation energies was then selected for the new parameter estimation, 
and for uncertainty analysis using the Monte Carlo method with Bootstraps sampling. The Bootstraps sampling was performed 200 times from the data residuals, (correlation matrix and sampling results available in Supporting information, tables S.4 and S.5 and figure E.1, Section E). The newly estimated values for the activation energies are shown in table 3 , together with the reported values from literature ${ }^{[29]}$, and the model outputs, confidence intervals and available experimental data are presented in figure 4.

The model output recreates with high precision the experimental data published by Seayad et al. ${ }^{[29]}$. With the exception of the end of reaction concentrations for the resulting products (IBU and IPPA, and IBS) most of the points are caught within the confidence interval of the carbonylation model.

\begin{tabular}{|c|c|c|c|c|c|}
\hline & & \multicolumn{2}{|c|}{ Chaudhari et al.[24] } & \multicolumn{2}{|l|}{ New estimation } \\
\hline & & Reported value & $\sigma$ & Estimated value & $\sigma$ \\
\hline \multirow{4}{*}{ 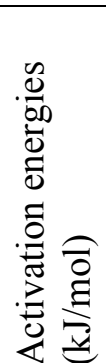 } & $E_{a 1}$ & 64.35 & - & 21.77 & 0.06 \\
\hline & $E_{a 2}$ & 49.49 & - & 43.62 & 0.40 \\
\hline & $E_{a-2}$ & 79.39 & - & 34.72 & 1.01 \\
\hline & $E_{a 3}$ & 89.08 & - & 33.86 & 0.10 \\
\hline
\end{tabular}


Dynamic simulation - Together with the hydrogenation step, carbonylation has been implemented into model flowsheet. IBAP is fed to the MIXER 1 unit with n-Decane and $\mathrm{Pd} / \mathrm{S}_{\mathrm{i}} \mathrm{O}_{2}$ as the solvent ${ }^{[28]}$. The final concentration of IBAP was set to be $0.27 \mathrm{~mol} / \mathrm{L}$ and the catalyst $0.5 \mathrm{~g} / \mathrm{L}$. For the tanks preceding the hydrogenation reactor (Tank Pre-Hydrogenation) the initial concentration is set to be the same as the cyclic steady-state feed, which in this case is the same as the outcome from the MIXER 1 unit. The tank is set with an initial holdup enough to cover a full upcoming operation. The same strategy is applied to remaining tanks, where the initial composition of these units that follow a reactor is the same as the expected from a cyclic-steady state (no disturbances). The hydrogenation reactor is set to the same operation conditions as reported by Thakar et al., with a constant hydrogen partial pressure of 20 bar, at constant temperature, $373 \mathrm{~K}$. It is assumed perfect mixing, and inexistent mass transfer limitations between gas-liquid. The reaction occurs for 25 minutes before emptying the reactor, re-filling, and repeating the process.

For the first separation process, it was assumed that the formed oligomers and catalyst were perfectly separated. As reported in table S.2 in the Supporting Information, the predicted boiling point of IBEB was the lowest on from the hydrogenation compounds. Thus it was assumed to be 
perfectly separable. A fixed recovery of $98 \%$ of IBPE was set, with $95 \%$ molar purity. The remaining fraction was divided according to the feed ration for the remaining components. This outlet is then mixed with a fresh feed of $\mathrm{MEK}, \mathrm{HCl}$, water and the second homogeneous catalyst $\left(\mathrm{PdCl} 2\left(\mathrm{PPh}_{3}\right)_{2} / \mathrm{PPh}_{3}{ }^{[29]}\right.$. The amount mixed ensures the pre-carbonylation IBPE concentration of $1.13 \mathrm{~mol} / \mathrm{L}$, water concentration of $2.67 \mathrm{~mol} / \mathrm{L}$, and $\mathrm{HCl}$ concentration of $0.24 \mathrm{~mol} / \mathrm{L}$. The carbonylation reactor operates at 54 bar, constant temperature of $388 \mathrm{~K}$, for 90 minutes. IBAP does not react within this operation.

The second separation step assumes a set of different steps, as aqueous/organic separation and distillations. For the aqueous/organic separation, it is assumed that the separation is ideal, and the remaining $\mathrm{HCl}$ and catalyst is removed together with the water from the process. The assumption for the second separation step is different than the separation process 1 . Three outlet streams are the result of this step, recreating a light Key stream, medium Key stream and heavy Key stream flows. Table 4 shows the outlet/feed ratio assumed for the different components in the outlet streams.

\begin{tabular}{|l|l|l|l|l|l|l|l|}
\hline \multicolumn{7}{|l|}{ Table 4-Assumed outlet ratio for the second separation step } \\
\hline $\begin{array}{l}\text { Outlet/feed } \\
\text { ratio }\end{array}$ & MEK & IBPA & IBAP & IBS & IBPCl & IBU & 3-IPPAP \\
\hline Light key & 1 & 0.998 & 0.998 & 1 & 0.999 & 0.02 & 0 \\
\hline Medium key & 0 & 0.002 & 0.002 & 0 & 0.001 & 0.96 & 0.20 \\
\hline Heavy key & 0 & 0 & 0 & 0 & 0 & 0.02 & 0.8 \\
\hline
\end{tabular}

The cooling crystallization operates for a total of 5 hours, with a starting temperature of 32 , a constant decrease until 28, and an end temperature of 11 . The time for the linear decrease of temperature $\left(T_{\text {linear }}\right)$ was set to be 30 minutes.

Figures 5, 6 and 7 show the cyclic steady state achieved for the upstream synthesis of Ibuprofen. The tank levels start in cyclic steady state. The difference between the minimum and maximum 
holdup level achieved for each one is due to the differences in filling and emptying time. This is due to the fact that one of the valves is always open, and continues to empty (in case of following a reactor) or continue to fill (for reactors preceding the reactors).

The profiles of the reactors mimic the results obtained from literature ${ }^{[28,29]}$. The results presented are shown in different time scales in order for better understanding and visualization. The steady concentration of the components within the end of each separated batch can be observed. During this time, the reactor is emptying, and the concentration values do not change. In contrast, when the filling process starts, there is an abrupt change in the concentrations due to the feed of solvent not containing any product (IBPE/IBEB for the hydrogenation step, and IBU/IPPAP for the carbonylation). After a brief stabilisation, the process restarts.
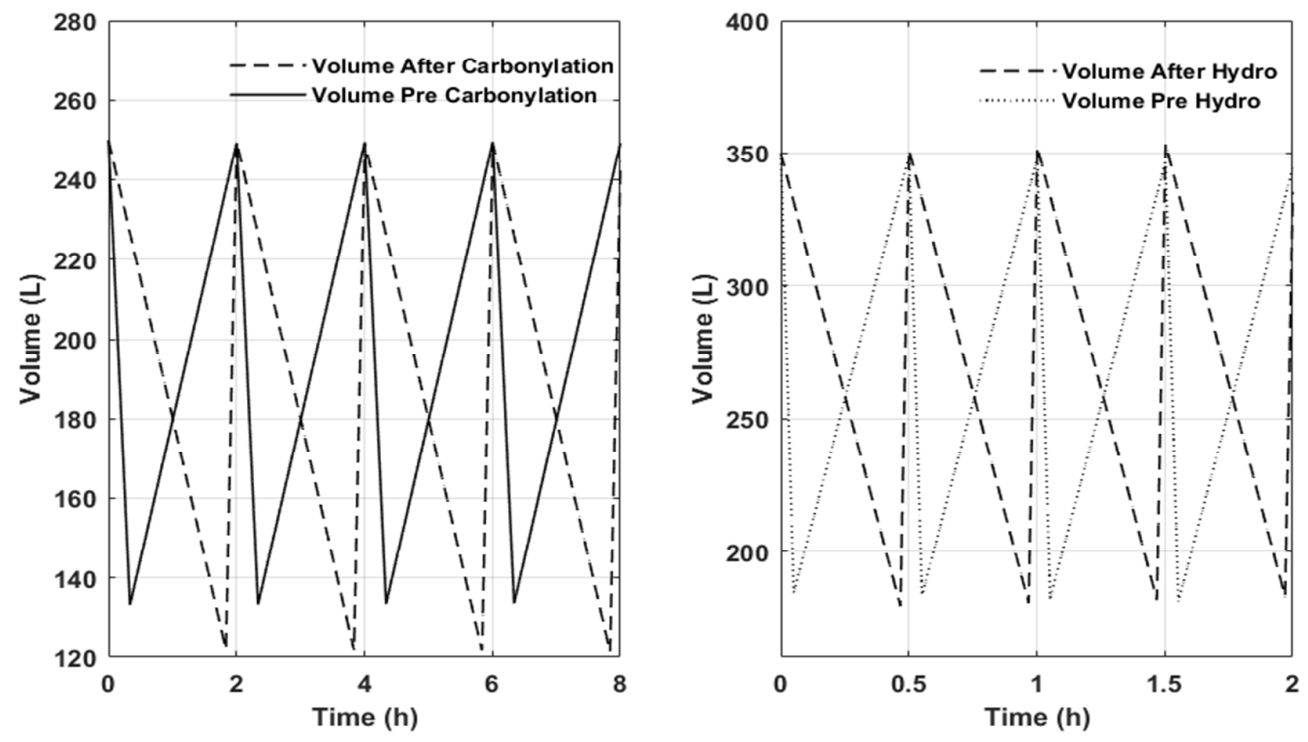

Figure 5 - Dynamic behaviour of the buffer tanks before and after the main reactions carbonylation (left) and hydrogenation (right) 

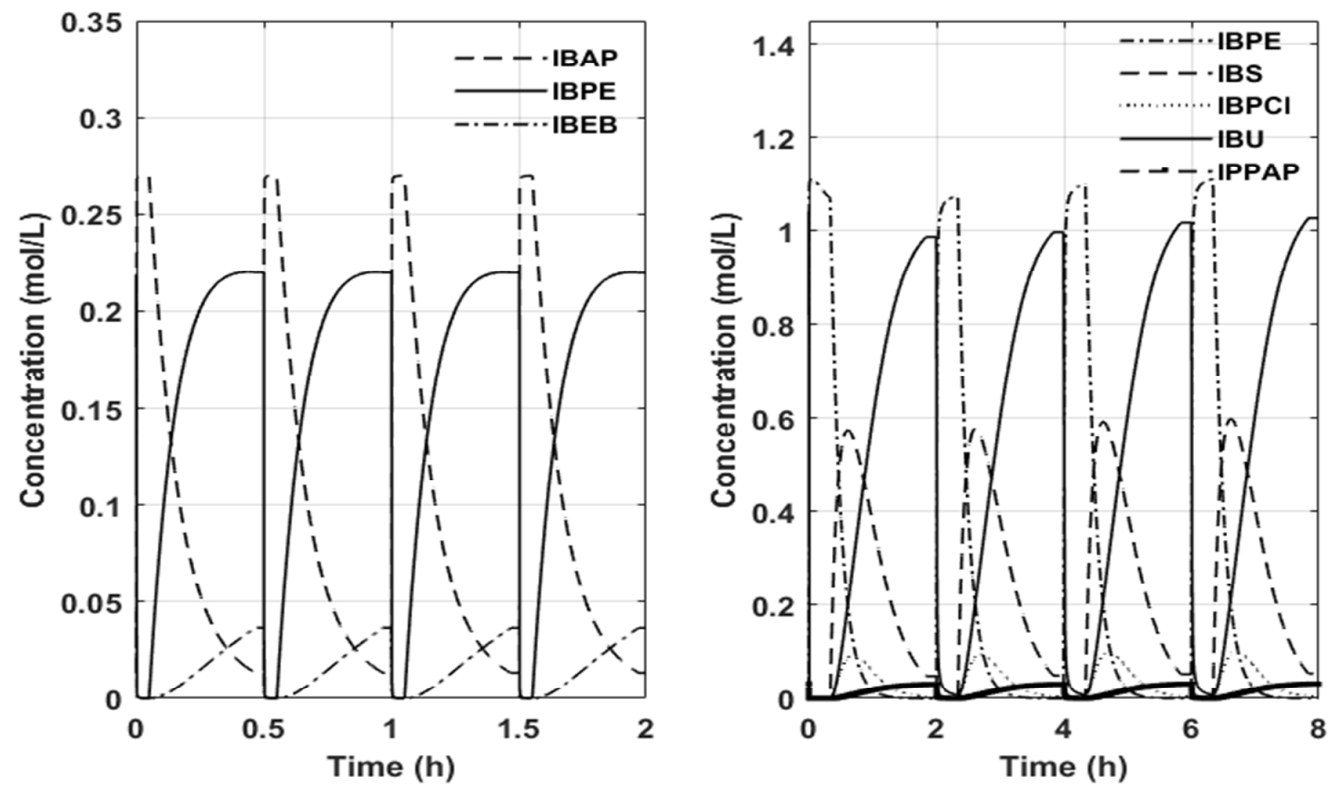

Figure 6 - Dynamic behaviour of hydrogenation and carbonylation reaction

The ibuprofen cooling crystallization occurs with secondary nucleation. As it can be observed after 5 hours of crystallization, a small amount of crystal is formed and accumulated near the first classes $\left(\mathrm{X}_{\text {size }}\right.$ and $\mathrm{Y}_{\text {size }}$ tending to zero). The effect of a small growth constant for ibuprofen crystal in ethanol/water concentration can be observed on figure 8 (left). Although the temperature closely follows the inlet temperature of the cooling serpentine, the gap between the saturation concentration and the solute slowly converges. 

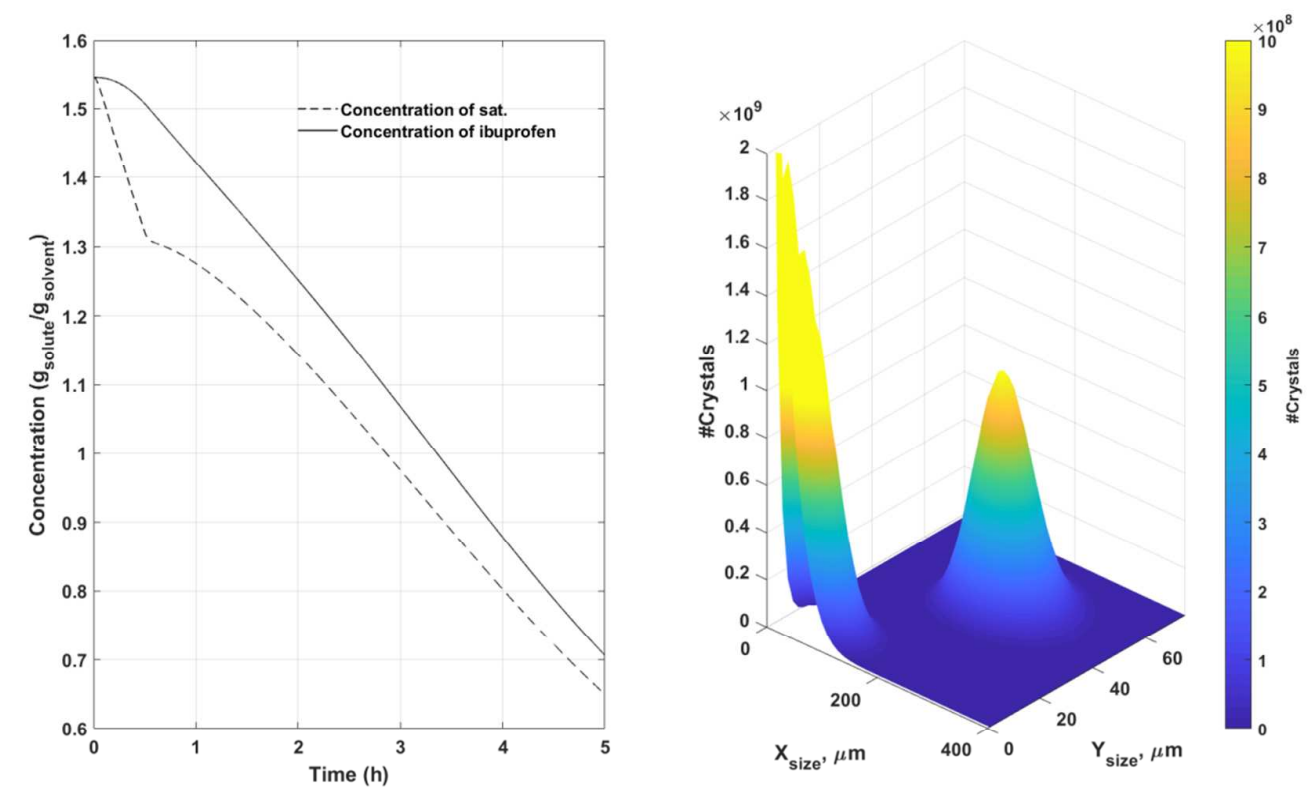

Figure 7 - Ibuprofen solute concentration and saturation profile over the 5 hours crystallization(left), and CSD of ibuprofen crystal after 5 hours crystallization (right).

The cyclic steady state obtained from the resulting simulation achieves a steady production of approximately $48.1 \mathrm{kgs}$ of ibuprofen per crystallization batch. The final solute concentration is approximately $0.70 \mathrm{~kg}_{\mathrm{ibu}} / \mathrm{kg}_{\text {solvent }}$, higher than the saturated concentration at $12^{\circ} \mathrm{C}$. This is due to the slow growth of the API. However, part of the crystalized API does not meet the requirements assumed for this process $(150 \mu \mathrm{m}$ for the $x$ characteristic length, and $70 \mu \mathrm{m}$ for the $y$ characteristic length). Roughly $3.14 \mathrm{kgs}$ of the total mass of crystal does not have the minimum size attributes to be considered valid for downstream processing.

Local sensitivity analysis - Figures 8 and 9 show the dynamic behaviour of the upstream synthesis of ibuprofen with continuous disturbances, on key process variables. These disturbances were introduced 5 minutes after the simulation start. The relative deviation to the initial case can be seen in table S.6, Supporting Information (section F). The effect of these disturbances propagates 
from process to process, as no control is implemented. It is also noticeable the time delay from batch to batch, as the whole process takes considerable time until reaching the new steady state. Changes in pressure and temperature are instantaneous in the respective reaction process, but are slowly propagated downstream through the process.

As the purity fractions were assumed and no data is provided related to the co-crystallization of IPPAP, no further analysis has been done regarding the ppm of this by-product in the crystallizer.
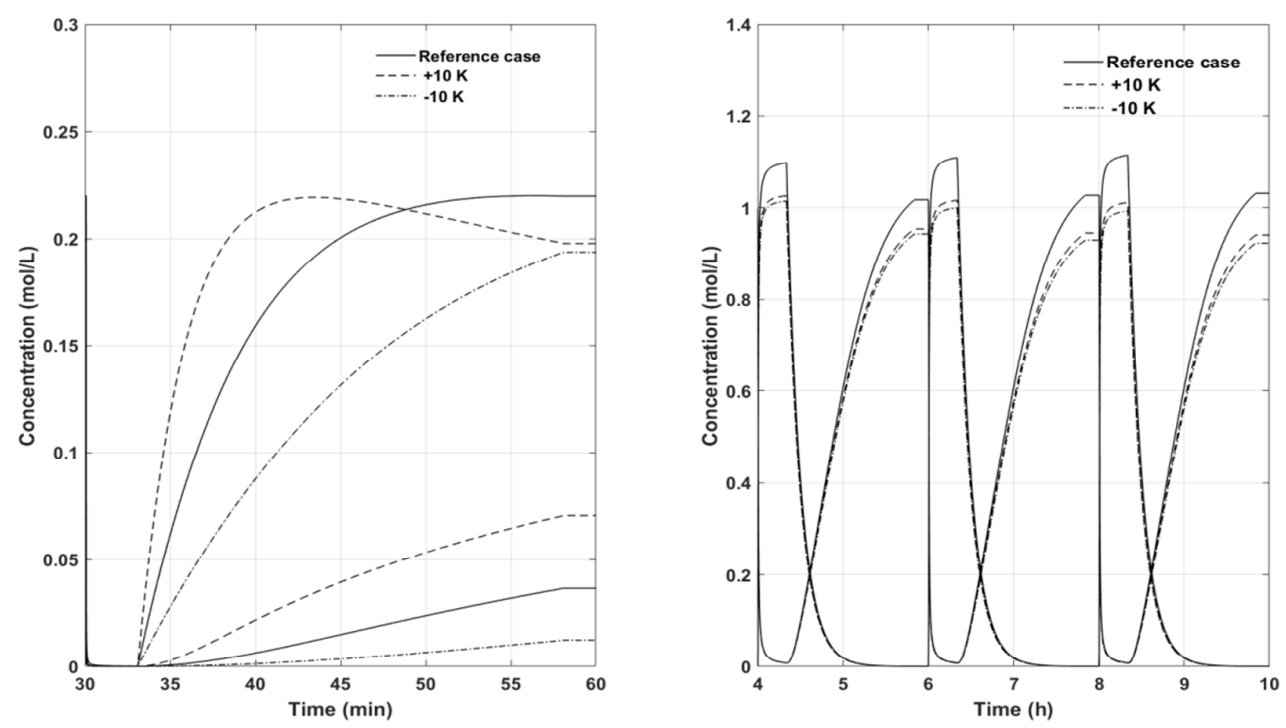

Figure 8 - Changes in IBPE and IBEB (left), and changes in IBPE and IBU (right) in response to temperature changes in hydrogenation reaction.

The most influential process variables are the temperatures. Within the range of 10 degrees, ibuprofen final concentration ranges from $1.7 \%$ to $-4.5 \%$ (based on reference case), per percent point variation within the temperature. This effect is peculiar in the hydrogenation temperature. Both changes decrease the overall synthesis of Ibuprofen, because the initial reagent IBPE is converted in higher amounts to the non-desired by-product, IBEB. 

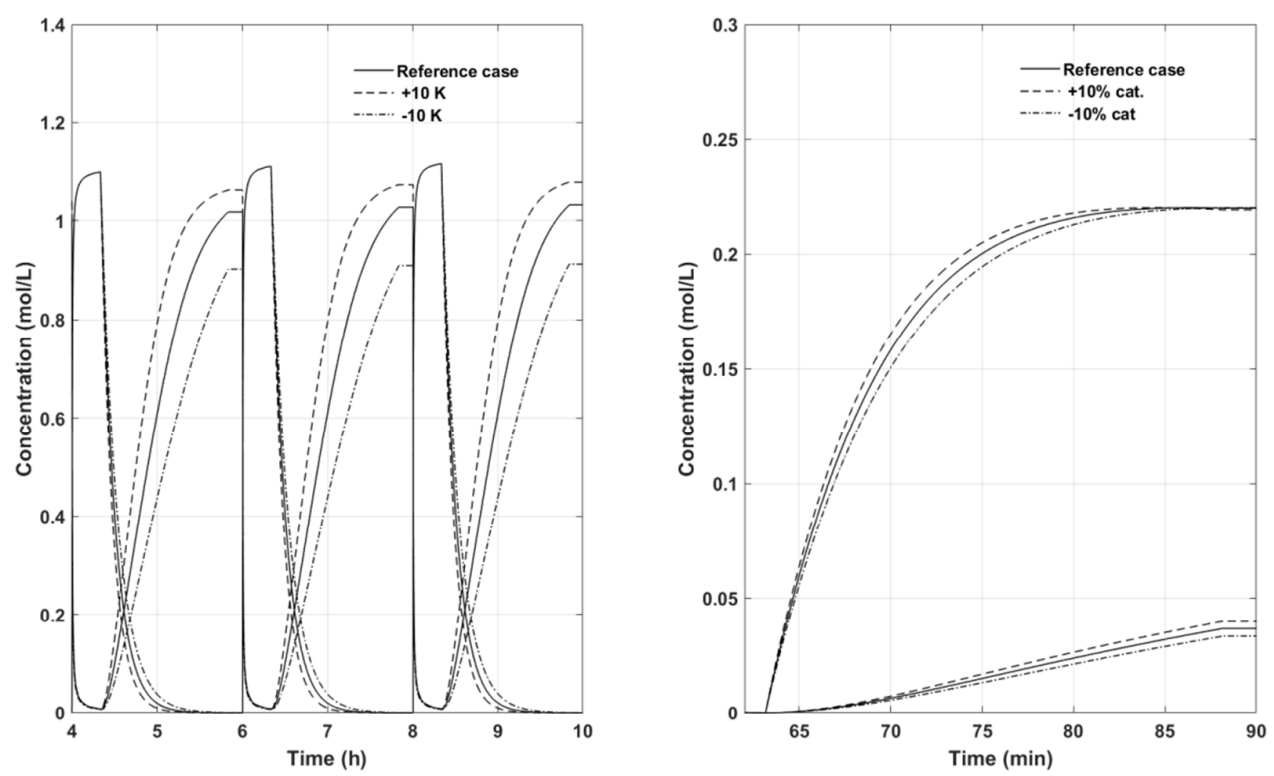

Figure 9 - Changes in IBPE and IBU concentration profiles in response to the temperature changes in carbonylation reaction (left) and in IBPE and IBEB due to catalyst concentration changes (right)

Figures 10 and 11 show the evolution of the main hydrogenation products (IBPE and IBEB) over time, over different temperature profiles. It can be observed that the hydrogenation step does not operate in its optimal temperature for the provided reaction time (25 minutes). However, this opens a space of decisions for a possible process engineer: it is possible to achieve the same or even higher final concentration of IBPE in lower time, while operating the process at higher temperatures. The downside is that the optimal region for stopping the reaction is smaller, and mistakes in the scheduling can propagate huge errors: lower final concentration of IBPE and higher undesired amounts of IBEB. On the other side, this time interval for stopping the reaction is bigger if the temperatures are lower. This comes with the cost of more operating time. 


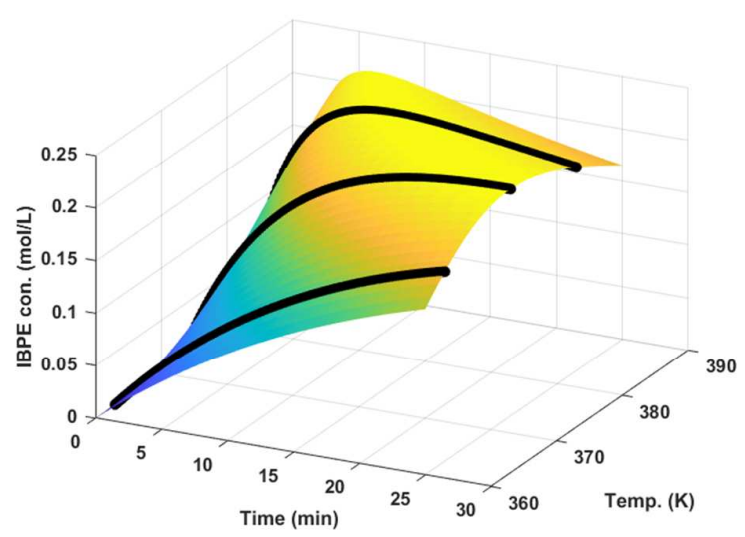

Figure 10 - Effect of temperature on IBPE concentration profiles over time, in the hydrogenation reaction. The dark lines represent the sensitivity cases simulated.

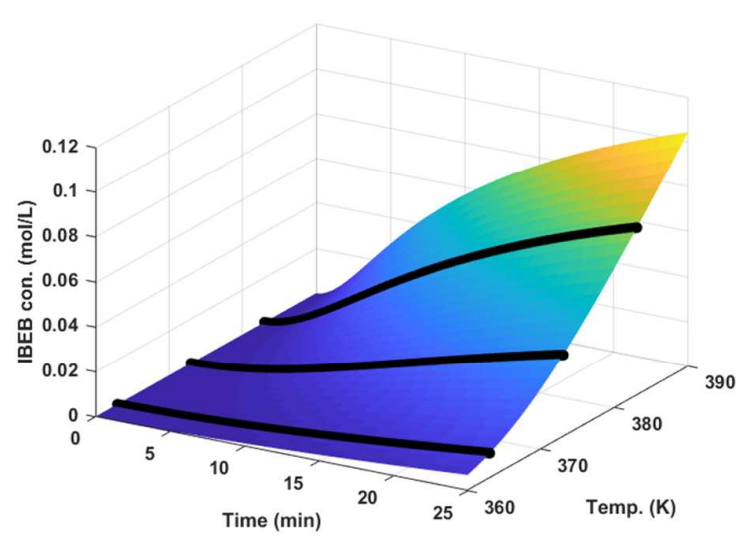

Figure 11 -Effect of temperature on IBEB concentration profiles over time, in the hydrogenation reaction. The dark lines represent the sensitivity cases simulated.

Global sensitivity analysis - With the deviation from the upstream processes, and uncertainty available from literature, Standardized Regression Coefficients (SRC) method was applied to the crystallization process. Monte Carlo coupled with Latin hypercube sampling was used for simulating the possible input space. The list of input uncertainties is shown in table 5. Although that water was considered to be totally separated from the carbonylation process, and carefully added 
before the crystallization, the sensitivity performed will take into account possible deviations, in preparation for further work. It was assumed that the uncertainty in water is huge, ranging from $90 \%$ less to $90 \%$ more from the initial case. Thus, the total mass of saturated solvent (aqueous ethanol), time duration of the linear decrease of temperature and water concentration were considered for the sampling process, as well as other parameters. For the sampling step, we performed 300 random samples using Latin hypercube sampling technique from multivariate input space, as defined in Table 5. The samples are shown in Supporting Information (section G).

\begin{tabular}{|c|c|c|c|}
\hline Parameter & Units & Initial value & Uncertainty range $(\%)^{[*]}$ \\
\hline Solvent mass $\left(m_{\mathrm{s}}\right)$ & $\mathrm{kg}$ & 55 & 15 \\
\hline Water concentration $\left(x_{\mathrm{w}}\right)$ & $\mathrm{kg} / \mathrm{kg}_{\text {solvent }}$ & 0.1 & 90 \\
\hline Linear cooling time $\left(t_{\text {linear }}\right)$ & $\min$ & 30 & 50 \\
\hline $\mathrm{k}_{\mathrm{b}}{ }^{[34]}$ & $\# / \mathrm{min} / \mathrm{kg}_{\mathrm{sol}}$ & 5.3 & 65 \\
\hline $\mathrm{kg}^{[32]}$ & $\mu \mathrm{m} / \mathrm{min} / \mathrm{Ssat}$ & $1.78 \mathrm{e}^{8}$ & 60 \\
\hline Initial seed mass $\left(m_{\text {seed }}\right)$ & $\mathrm{kg} / \mathrm{kg}_{\text {solvent }}$ & 0.05 & 50 \\
\hline
\end{tabular}

The impact of the specified input uncertainty on the output is calculated on three different points of the CSD (on the average $x$ characteristic length, $\mathrm{x}_{1}, \mathrm{x}_{2}$ and $\mathrm{x}_{3}$ ), chosen based on the simulations output (figure 13). This choice was based on the fact that $\mathrm{x}_{1}(65 \mu \mathrm{m})$ is a noticeable point of influence from the effect of the secondary nucleation, opposing to $\mathrm{x}_{3}(255 \mu \mathrm{m})$ that is dependent on both growth and initial API dissolved. The point $\mathrm{x}_{2}(185 \mu \mathrm{m})$ is within the range of the most 
probable mean characteristic size. In addition, the influence of the uncertainty is also taken in to consideration on the total mass of crystal formed, $T_{\text {mass }}$, and on the ratio of API below the desired size specifications, $r_{\text {low_spec. }}$

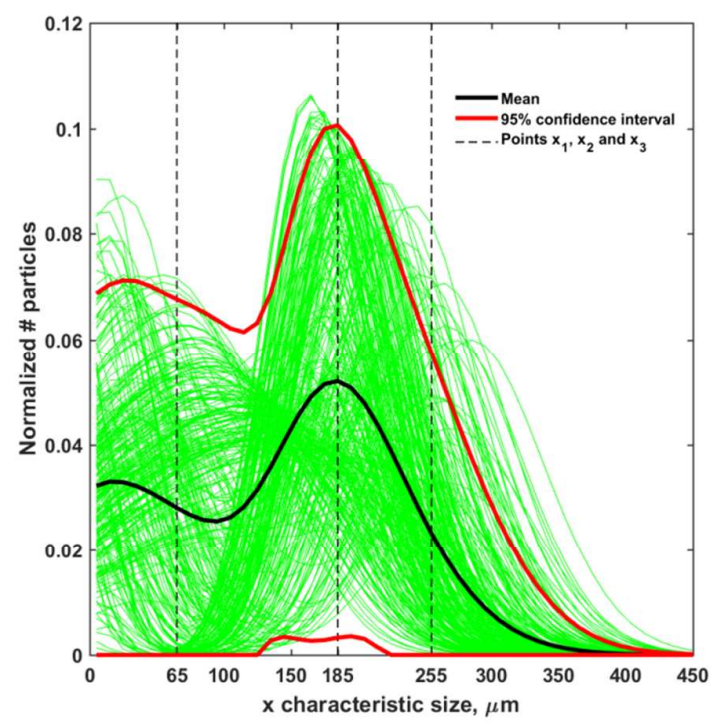

Figure 13- Representation of the uncertainty in the crystal size distribution

The linearization model obtained for the data on the three chosen points, $A P I_{\text {mass }}$ and $r_{\text {low_spec }}$ verifies applicability of SRC method, as coefficient of determination is sufficiently high, $R^{2}>0.7$ ${ }^{[42,43]}$. As it is shown in table 6 , the parameters have different significance depending on which output is observed. Regarding the average population in the chosen crystal lengths, the water concentration, the initial seed and the crystal growth parameter are the most influential. As water influences both the growth and birth of the ibuprofen crystals in the solvent, the population of crystals with $65 \mu \mathrm{m}$ will not increase with the decrease of growth and birth. This is due to the fact that secondary crystallization will yield a smaller population. In addition, the overall growth of crystals will be slower due to the water influence, and the small population formed will not reach the threshold of 65 before the crystallization is finished. The growth parameter, on the other hand, 
shifts the final CSD to bigger sizes, reducing the final amount of particles within the range of $\mathrm{x}_{1}$. This works in the opposite way for the other points $\left(\mathrm{x}_{2}\right.$ and $\left.\mathrm{x}_{3}\right)$. The amount of initial seed has an effect in how wide the crystal distribution will be. It has indeed an effect in the total number of crystal formed, however the highest influence is on the ratio of crystal underspecified. As more initial crystal (seed) is present in the medium, more solute can deposit and grow into bigger crystals. However, a huge number of seed will reduce the average growth, as the probability of a particle to deposit into a grown crystals is smaller, due to the huge number or particles. This is captured by the sensitivity analysis in the third point $\left(\mathrm{x}_{3}\right)$, where a positive change in the initial feed of crystal has a negative outcome on the bigger crystal classes.

In general, $k_{b}$ parameter has low impact on the specs of the different (points $\mathrm{x}_{1}, \mathrm{x}_{2}, \mathrm{x}_{3}$ ). For the smaller ones $\left(\mathrm{x}_{1}\right)$, the impact is positive, as the increase in number of newly born particles increases the chances that some will reach this target size. For bigger crystals, however, this can be understood as competition for solute deposition (thus, growth). It can be also observed that changing the initial fast decrease of temperature has no effects in the long run for this process. Enhancing or delaying the secondary nucleation has shown to have no impact in the final results. Therefore the influence of $\mathrm{t}_{\text {linear }}$ can be mostly neglected.

Lastly, by propagating the uncertainty shown above (table 5), the final mass of Ibuprofen crystalized has an average of $48.3 \mathrm{~kg}$ per batch unit, with a standard deviation of $4.4 \mathrm{~kg}$. The main case scenario is within these values, as well within the target specifications of the crystals, with an average of $11.6 \%$ mass lost, and a standard deviation of $9 \%$. 


\begin{tabular}{|c|c|c|c|c|c|c|c|c|}
\hline \multicolumn{9}{|c|}{ Table 6 - Standardized regression coefficients and parameter significance ranking } \\
\hline Output & $R^{2}$ & Ranking & 1 & 2 & 3 & 4 & 5 & 6 \\
\hline \multirow{2}{*}{$r_{\text {low_spec }}$} & \multirow{2}{*}{0.811} & Par. & $m_{\text {seed }}$ & $k_{g}$ & $x_{\mathrm{w}}$ & $m_{\mathrm{s}}$ & $k_{b}$ & $t_{\text {linear }}$ \\
\hline & & $\beta_{\mathrm{i}, \mathrm{j}}$ & 0.64 & -0.46 & 0.31 & -0.19 & 0.14 & 0.03 \\
\hline \multirow{2}{*}{$\mathrm{x}_{1}$} & \multirow{2}{*}{0.815} & Par. & $k_{g}$ & $x_{\mathrm{w}}$ & $m_{\text {seed }}$ & $k_{b}$ & $m_{\mathrm{s}}$ & $t_{\text {linear }}$ \\
\hline & & $\beta_{\mathrm{i}, \mathrm{j}}$ & -0.71 & -0.42 & 0.32 & 0.19 & 0.11 & 0.08 \\
\hline \multirow{2}{*}{$\mathrm{x}_{2}$} & \multirow{2}{*}{0.703} & Par. & $m_{\text {seed }}$ & $k_{g}$ & $x_{\mathrm{w}}$ & $k_{b}$ & $m_{\mathrm{s}}$ & $t_{\text {linear }}$ \\
\hline & & $\beta_{i, j}$ & 0.51 & 0.48 & 0.41 & -0.24 & -0.12 & -0.06 \\
\hline \multirow{2}{*}{$x_{3}$} & \multirow{2}{*}{0.757} & Par. & $k_{g}$ & $x_{\mathrm{w}}$ & $m_{\text {seed }}$ & $k_{b}$ & $m_{\mathrm{s}}$ & $t_{\text {linear }}$ \\
\hline & & $\beta_{i, j}$ & 0.59 & -0.42 & 0.42 & -0.13 & 0.10 & -0.06 \\
\hline \multirow{2}{*}{$A P I_{\text {mass }}$} & \multirow{2}{*}{0.967} & Par. & $m_{\mathrm{s}}$ & $k_{g}$ & $m_{\text {seed }}$ & $x_{\mathrm{w}}$ & $t_{\text {linear }}$ & $k_{b}$ \\
\hline & & $\overline{\beta_{i, j}}$ & 0.85 & 0.35 & 0.25 & 0.17 & -0.04 & 0.03 \\
\hline
\end{tabular}

\section{Conclusions}

An upstream model-based synthesis of Ibuprofen, based on the Hoescht pathway, has been presented. The models available for unit operations have been validated using the available open literature experimental data, and improved further following a systematic and comprehensive identifiability and uncertainty analysis, which provided new parameter values with lower prediction uncertainty.

The cyclic steady state is achieved with success within the model parameters and the assumptions made. The crystallization of approximately $48 \mathrm{~kg}$ of ibuprofen is achieved within each cycle. 93 percent of the crystals (weight based) conformed with the target specifications of the crystals - 
defined as being smaller than $150 \mu \mathrm{m}$ length, and $70 \mu \mathrm{m}$ width. Thus, per cycle, $3.4 \mathrm{~kg}$ of the API are not taken into account for further downstream processing.

It has been shown that the most influential process parameters are the operating temperatures in both reactors. While greatly influencing the target ibuprofen production and by-products synthesis, further work needs to be done regarding the difficulty of separating the by-products, within their wide design space. Future work will focus on detailed modelling of separation processes and propagation of impurities from recovery step to purification of ibuprofen.

The Monte Carlo sensitivity analysis performed in the crystallization unit has shown that the uncertainty provided in literature has a huge impact in the whole possible outcome of this process. Furthermore, one of the most uncertain parameters, $k_{g}$, is also one of the most influential attributes in the spectrum of the CSD and total API produced. Other influential parameters include the initial seed mass, and water concentration in the process. These parameters can easily be controlled, and indeed current work is focusing on the development and validation of a nonlinear control strategy on laboratory scale to reject the identified process disturbances on ibuprofen crystallisation process.

Overall the dynamic flowsheet modelling platform is demonstrated as a valuable enabling tool to systematically integrate knowledge about different unit operations in a form of model, perform systematic and comprehensive error propagation as well as sensitivity and uncertainty analysis for verifying the robustness of pharmaceutical manufacturing processes.

\section{Supporting Information}

Additional content and tables as noted in the text. This information is available free of charge via internet at http://pubs.acs.org/. 


\section{Acknowledgments}

This work has received funding from the European Union's Horizon 2020 research and innovation programme under the Marie Sklodowska-Curie grant agreement no. 675251.

\section{References}

[1] - Sheldon, R. A. The E factor: Fifteen years on. Green Chemistry, 2007, 9, 1273-1283

[2] - Gernaey, K. V.; Cervera-Padrell, A. E.; Woodley, J. M.; A perspective on PSE in pharmaceutical process development and innovation, Computers and Chemical Engineering, 2012, $42,15-29$

[3] - DiMasia, J. A.; Hansenb, R. W.; Grabowskic, H. G. The price of innovation: New estimates of drug development costs. J. Health Econ. 2003, 22 (2), 151-185.

[4] - Basu, P.; Joglekar, G.; Rai, S.; Suresh, P.; Vernon, J. Analysis of manufacturing costs in pharmaceutical companies. J. Pharm. Innov. 2008, 3, 30-40.

[5] - FDA; Innovation, stagnation: Challenges and opportunity on the critical path to new medical products. Food and Drug Administration: Washington, DC, 2004

[6] - FDA; PAT - A framework for innovative Pharmaceutical Development, Manufacturing and Quality Assurance, Pharmaceutical CGMPs, 2004

[7] - Kourti, T.; Process analytical technology beyond real-time analyzers: The role of multivariate analysis. Critical Reviews in Analytical Chemistry, 2006, 36, 257-278.

[8] - Mears, L.; Stocks, S.; Albæk, M. O.; Sin, G.; Gernaey, K. V.; Mechanistic Fermentation Models for Process Design, Monitoring and Control. Trends in Biotechnology, 2017, 10, 914-924 
[9] - Linniger, A. A.; Stephanopoulos, E.; Ali, S. A.; Han, C.; Stephanopoulos, G.; Generation and Assessment of Batch Processes with Ecological Considerations; Computers Chemical Engineering, 1995, vol 19,pg 7-13

[10] - Linniger, A. A.; Ali, S. A.; Stephanopoulos, G.; Knowledge-Based validation and waste management of batch pharmaceutical process designs; Computers Chemical Engineering; 1996, vol 20 , pg 1431-1436

[11] - Chakraborty, A; Linninger, A. A.; Plant-Wide Management. 1 Synthesis and Multiobjective Design; Ind. Eng. Chem. Res.; 2002; vol 41, pg 4592 - 4604.

[12] - Benyahia, B.; Lakerveld, R.; Barton, P. I.; A Plant-Wide Dynamic Model of a Continuous Pharmaceutical Process. Industrial \& Engineering Chemistry Reasearch, 2012, 51, 15393 - 15412.

[13]- Schaber, S. D.; Gerogiorgis, D. I.; Ramachandran, R.; Evans, J. M. B.; Barton, P. I.; Trout, B. L. Economic analysis of integrated continuous and batch pharmaceutical manufacturing: A case study, Ind. Eng. Chem. Res. 2011, 50, 10083-10092.

[14]- Jolliffe, H. G.; Gerogiorgis, D. I.; Plantwide design and economic evaluation of two Continuous Pharmaceutical Manufacturing (CPM) cases: Ibuprofen and Artemisinin, Computers and Chemical Engineering, 2016, 91, 269-288

[15]- Sin, G., Gernaey, K. V., Eliasson Lantz, A.; Good modelling practice (GMoP) for PAT applications: Propagation of input uncertainty and sensitivity analysis. Biotechnology Progress, 2009, 25, 1043-1053.

[16]- Paakkunainen, M., Matero, S., Ketolainen, J., Lahtela-Kakkonen, M., Poso, A., Reinikainen, S. P.; Uncertainty in dissolution test of drug release, Chemometrics and Intelligent Laboratory Systems, 2009, 97, 82-90. 
[17]- Gargalo, C. L.; Cheali, P.; Posada, J. A.; Gearnaey, K. V.; Sin, G.; Economic Risk Assessment of Early Stage Designs for Glycerol Valorization in Biorefinery Concepts, Industrial \& Engineering Chemistry, 2016, 55, 6891-6814.

[18] - Chakraborty, A; Linninger, A. A.; Plant-Wide Waste Management 2. Decision Making under Uncertainty; Ind. Eng. Chem. Res.; 2003; vol 42, pg 357-369

[19] - Chakraborty, A; Colberg, R. D.; Linninger, A. A.; Plant-Wide Waste Management 3. LongTerm Operation and Investment Planning under Uncertainty; Ind. Eng. Chem. Res.; 2003; vol 42, pg $4772-4788$

[20] - Franceschini, G.; Macchietto, S.; Model-based design of experiments for parameter precision: State of the art. Chemical Engineering Science, 2008, 63, 4846-4872.

[21]- Singh, R., Sahay, A., Karry, K. M., Muzzio, F., Iarapetritou, M., Ramachandran, R.; Implementation of an advanced hybrid MPC-PID control system using PAT tools into direct compaction continuous pharmaceutical tablet manufacturing pilot plant, International Journal of Pharmaceuticals, 2014, 473, 38-54.

[22] - Nayhouse, M., Tran, A., Kwon, J. S., Crose, M., Orkoulas, G., Christofides, P.,D., Modeling and control of ibuprofen crystal growth and size distribution, Chemical Engineering Science, 2015, 134, 414-42.

[23]- Shi, D., El-Farra, N. H., Li, M., Mhaskar, P., Christofides, P. D., Predictive control of particle size distribution in particulate processes, Chemical Engineering Science, 2006, 61, 268-261

[24]- Nagy, Z. K., Fujiwara, M., Braatz, R. D., Modelling and control of combined cooling and antisolvent crystallization processes. Journal of Process Control, 2008, 18, 856-864. 
[25]- Adams, S.S., The propionic acids: a personal perspective. J. Clin. Pharmacol. 1992, 32(4): $317-323$

[26] - Bogdan, A.R., Poe, S.L., Kubis, D.C. Broadwater, S. J., McQuade, D. T., The continuousflow synthesis of ibuprofen. Angeandte Chemie International Edition, 2009, 48(45), 8547-8550

[27]- Thakar, N., Schildhauer T., Buijs W., Kapteijn F., Moulijn J., Evaluation of deactivation mechanisms of Pd-catalyzed hydrogenation of 4- isobutylacetophenone, Journal of Catalysis, 2007, 248(2), 249-257

[28] - Thakar, N., Berger R. J., Kapteijn F., Moulijn J., Modelling kinetics and deactivation for the selective hydrogenation of an aromatic ketone over $\mathrm{Pd} / \mathrm{SiO} 2$, Chemical Engineering Science, 2007, 62, 5322-5329

[29]- Seayad, A. M., Seayad, J., Mills, P. L., Chaudhari R. V., Kinetic Modelling of Carbonylation of 1-(4-Isobutylphenyl)ethanol using a homogeneous $\mathrm{PdCl}_{2}\left(\mathrm{PPh}_{3}\right)_{2} / \mathrm{T}_{\mathrm{s}} \mathrm{OH} / \mathrm{LiCl}$ Catalyst System, Ind. Eng. Chem Res., 2003, 42, 2496-2506

[30]- Elango, V., Murphy, M. A., Smith, B. L., Davenport, G. K., Mott, G. N., Zey, E. G., Moss, G. L., Method for producing Ibuprofen, US 4981995 A, https://www.google.com/patents/

[31]- Samad, N. A. F. B. A., Control of Process Operations and Monitoring of product Qualities through Generic Model-based Framework in Crystallization Processes, Ph.D. Thesis, Technical University of Denmark, 2012

[32]- Rashid, A., White, E. T., Howes, T., Litster, J. D., Marziano, I., Growth rates of ibuprofen crystals grown from ethanol and aqueous ethanol, Chemical Engineering Research and Design, 2012, 90, 158-161. 
[33]- Rashid, A., White, E. T., Howes, T., Litster, J. D., Marziano, I., Metastable zone width for racemic ibuprofen in ethanol and aqueous ethanol mixtures, Proc. Chemeca, Perth, 2009, 118

[34] - Rashid, A., White, E. T., Howes, T.,Liu, L., Litster, J. D., Marziano, I., Nucleation kinetics for ibuprofen crystals grown from aqueous ethanol, Proc.Cchemeca, Sydney, 2011, 107

[35]- Nguyen, T. T. H., Hammond, R. B., Marziano, I., Nichols, G., Precision measurement of the growth rate and mechanism of ibuprofen 001 and 011 as a function of crystallization environment, Cryst. Eng. Community, 2014, 16, 4568-4586.

[36] - Sin, G., Meyer, A. S., Gernaey, K. V., Assessing reliability of cellulose hydrolusis models to support biofuel process design -Identifiability and Sensitivity Analysis; Computers and Chemical Engineering; 2010, 34, 1385-1392

[37] - Seber, G., Wild, C.; Nonlinear regression; 1989, New York: Wiley

[38] - Brun, R., Kuhni, M., Siegrist, H., Gujer, W., Reichert, P., Practical identifiability of ASM2d parameters - systematic selection and tuning of parameter subsets, Water Research, 2002, $36,4113-4127$

[39]- Efron, B., Bootstrap methods. Another look at the jackknife, The annals of Statistics, 1979, $7,1-26$

[40]- Frutiger, J., Marcarie, C., Abildskov, J., \& Sin, GA Comprehensive Methodology for Development, Parameter Estimation, and Uncertainty Analysis of Group Contribution Based Property Models- An Application to the Heat of Combustion. Journal of Chemical \& Engineering Data, 2015, 61(1), 602-613. 
2

3

4

5

6

7

8

9

10

[41]- Jones, M. N., Hukkerikar, A., Sin, G., Gani, R., Sensitivity of process design to uncertainties in property estimates applied to extractive distillation, Chisa, Prague, $\mathbf{2 0 1 2}$

[42] -McKay, M.D., Beckman, R. J., Conover W. J.; A comparison of three methods for selecting values of input variables in the analysis of output from computer code; Technometrics; 1979; 21; 239-245

[43]- Campolongo, F., Saltelli, A., Sensitivity analysis of an environmental model: an application of different analysis methods, Reliability Engineering and System Safety, 1997, 57, 49-69 


\section{For Label of Contents Only}

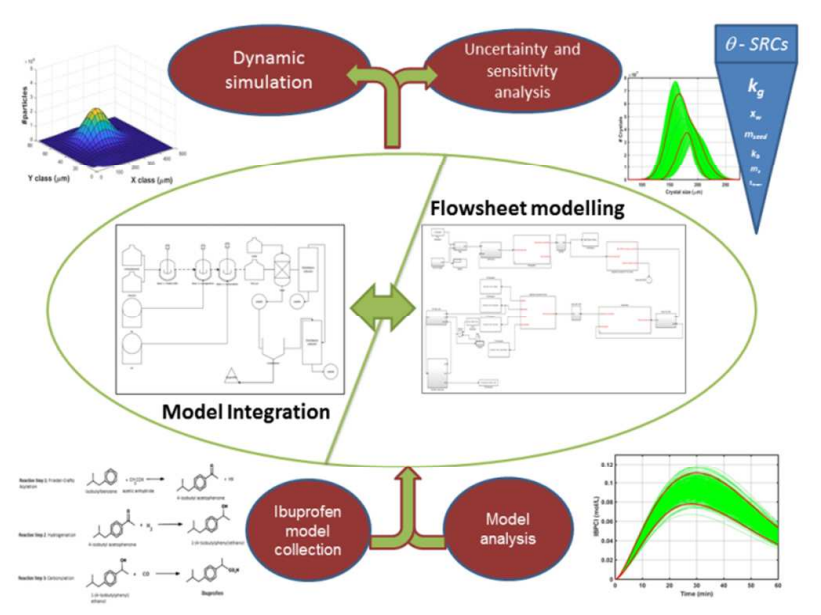

30 